\title{
Rituximab Treatment in a Patient With Primary Biliary Cirrhosis and Rheumatoid Arthritis
}

\author{
Betül SARGIN, Gülcan GÜRER \\ Department of Physical Medicine and Rehabilitation, Medical Faculty of Adnan Menderes University, Aydin, Turkey
}

Primary biliary cirrhosis (PBC) is an autoimmune liver disease, ${ }^{1}$ with symptoms including fatigue, cognitive impairment, and pruritus. ${ }^{2}$ Rheumatoid arthritis (RA) is a chronic inflammatory autoimmune disease characterized by joint destruction and disability. ${ }^{3}$ Herein, we report a female patient with $\mathrm{PBC}$ and RA successfully treated with rituximab.

A 72-year-old female patient who had been diagnosed with $\mathrm{PBC}$ for three years complaining of pain, swelling, and warmth in her wrists and ankles, with accompanying morning stiffness lasting for one hour for one year applied to our outpatient clinic. The diagnosis of PBC was considered upon two of three objective criteria: (i) biochemical evidence of cholestasis based on elevated level of serum alkaline phosphatase (1.5 times the upper limit of normal); (ii) presence of serum antimitochondrial antibodies (titers of 1:40); and (iii) liver histology characterized by nonsuppurative cholangitis and interlobular bile duct destruction. ${ }^{4}$ She was taking ursodeoxycholic acid for PBC treatment. Tenderness and warmth were detected in both wrists and ankles in musculoskeletal system examination. Patient's laboratory findings are presented in Table 1. X-rays of wrists and ankles were consistent with RA. Following RA diagnosis, treatment with oral sulfasalazine ( $2 \mathrm{~g}$ /day), hydroxychloroquine (400 mg/day), and prednisolone $(10 \mathrm{mg} /$ day $)$ was initiated. After refractoriness to synthetic disease-modifying antirheumatic drugs (Disease Activity Score 28: 6.96) and tumor necrosis factor alpha inhibitor therapies (etanercept and adalimumab), patient received rituximab cycles with two infusions every six months. Response to treatment was assessed by Disease Activity Score 28. After the second cure, Disease Activity Score 28 decreased to 4.5 , and serum alkaline phosphatase and gamma glutamyl transferase levels regressed to $82 \mathrm{U} / \mathrm{L}$ and $23 \mathrm{U} / \mathrm{L}$, respectively. Her symptoms regressed.

The prevalence of the association of $\mathrm{PBC}$ and $\mathrm{RA}$ is $1.8 \%$ to $5.6 \% .^{5} \mathrm{~B}$ cells may have important role in the pathogenesis of these diseases. Tsuda et al. ${ }^{6}$ and Jopson et al. ${ }^{2}$ suggested rituximab as a successful treatment for PBC. In case reports by Lazrak et al. ${ }^{1}$ and Polido-Pereira et al., ${ }^{7}$ patients had good clinical and laboratory responses with rituximab treatment for $\mathrm{RA}$ although abnormalities in liver tests were persistent after five months and one year, respectively.

Although response to rituximab treatment have been reported differently in $\mathrm{PBC}$ and $\mathrm{RA}$ patients in the literature, our case suggests rituximab as a successful treatment option for these diseases supported with good responses in clinical and laboratory parameters.

In conclusion, rituximab treatment is an effective treatment option for patients with PBC and RA. 
Table 1. Laboratory findings of patient

\begin{tabular}{lcc}
\hline & Values & Normal range \\
\hline Hemoglobin (gr/dL) & 10.3 & $11.7-15.5$ \\
Hematocrit (\%) & 31.3 & $37-44$ \\
Mean corpuscular volume (fL) & 91.8 & $80.4-95.9$ \\
Red cell distribution width & 14.4 & $11.7-14.6$ \\
Leukocyte count (mkrL) & 8.68 & $3.800-11.000$ \\
Platelet (mkrL) & 383.000 & $150.000-350.000$ \\
Erythrocyte sedimentation rate (mm/h) & 90 & $0-20$ \\
C-reactive protein (mg/L) & 21.73 & $0-6$ \\
Urea (gr/dL) & 37 & $13-43$ \\
Creatinine (mg/dL) & 0.72 & $0.7-1.3$ \\
Alanine aminotransferase (IU/L) & 14 & $0-55$ \\
Alkaline phosphatase (U/L) & 302 & $40-150$ \\
Gamma glutamyl transpeptidase test (U/L) & 86 & $9-36$ \\
Rheumatoid factor (IU/mL) & 484.58 & $0-18$ \\
Anti-cyclic citrullinated peptide (U/mL) & 64.5 & $0-2.5$ \\
Antinuclear antibodies & Negative \\
Antimitochondrial antibodies & $1 / 100$ Positive & Negative \\
Anti-smooth muscle antibody & Negative & Negative \\
Brucella agglutination & Negative & Negative \\
\hline
\end{tabular}

\section{Declaration of conflicting interests}

The authors declared no conflicts of interest with respect to the authorship and/or publication of this article.

\section{Funding}

The authors received no financial support for the research and/or authorship of this article.

\section{REFERENCES}

1. Lazrak F, Abourazzak FE, Berrada K, Kadi N, Manssouri S, Harzy T. A rare association of rheumatoid arthritis and primary biliary cirrhosis treated with rituximab: a case report. J Med Case Rep 2013;7:99.

2. Jopson L, Newton JL, Palmer J, Floudas A, Isaacs J, Qian J, et al. RITPBC: B-cell depleting therapy (rituximab) as a treatment for fatigue in primary biliary cirrhosis: study protocol for a randomised controlled trial. BMJ Open 2015;5:7985.

3. Smolen JS, Aletaha D. Rheumatoid arthritis therapy reappraisal: strategies, opportunities and challenges. Nat Rev Rheumatol 2015;11:276-89.

4. Bowlus CL, Gershwin ME. The diagnosis of primary biliary cirrhosis. Autoimmun Rev 2014;13:441-4.

5. Marasini B, Gagetta M, Rossi V, Ferrari P. Rheumatic disorders and primary biliary cirrhosis: an appraisal of 170 Italian patients. Ann Rheum Dis 2001;60:1046-9.

6. Tsuda M, Moritoki Y, Lian ZX, Zhang W, Yoshida K, Wakabayashi $\mathrm{K}$, et al. Biochemical and immunologic effects of rituximab in patients with primary biliary cirrhosis and an incomplete response to ursodeoxycholic acid. Hepatology 2012;55:512-21.

7. Polido-Pereira J, Rodrigues AM, Canhão H, Saraiva F, da Silva JA, Fonseca JE. Primary biliary cirrhosis in a rheumatoid arthritis patient treated with rituximab, a case-based review. Clin Rheumatol 2012;31:385-9. 